\title{
Vav1: A Dr. Jekyll and Mr. Hyde protein - good for the hematopoietic system, bad for cancer
}

\author{
Shulamit Katzav ${ }^{1}$ \\ ${ }^{1}$ Developmental Biology and Cancer Research, IMRIC, Hebrew University-Hadassah Medical School, Jerusalem, Israel \\ Correspondence to: \\ Shulamit Katzav-Shapira, e-mail: shulamitk@ekmd.huji.ac.il
}

Keywords: Vav1, Rac, GEF, RhoGTPases, Cancer

Received: June 21, 2015

Accepted: August 07, 2015

Published: August 20, 2015

\section{ABSTRACT}

\begin{abstract}
Many deregulated signal transducer proteins are involved in various cancers at numerous stages of tumor development. One of these, Vav1, is normally expressed exclusively in the hematopoietic system, where it functions as a specific GDP/GTP nucleotide exchange factor (GEF), strictly regulated by tyrosine phosphorylation. Vav was first identified in an NIH3T3 screen for oncogenes. Although the oncogenic form of Vav1 identified in the screen has not been detected in clinical human tumors, its wild-type form has recently been implicated in mammalian malignancies, including neuroblastoma, melanoma, pancreatic, lung and breast cancers, and B-cell chronic lymphocytic leukemia. In addition, it was recently identified as a mutated gene in human cancers of various origins. However, the activity and contribution to cancer of these Vav1 mutants is still unclear. This review addresses the physiological function of wild-type Vav1 and its activity as an oncogene in human cancer. It also discusses the novel mutations identified in Vav1 in various cancers and their potential contribution to cancer development as oncogenes or tumor suppressor genes.
\end{abstract}

\section{INTRODUCTION}

The past few decades have witnessed a major leap in understanding of the molecular mechanisms involved in tumor pathogenesis and progression [1]. Signaling molecules that play critical roles in cancer were identified and served as targets for therapeutic drugs. For instance, the first drug targeting a cancer gene, Herceptin, is helpful for many breast cancer patients with tumors driven by the target hormone receptor, HER2 [2]. Another example is the drug Gleevec, used for inhibition of protein kinase $\mathrm{Abl}$ in Chronic Myelogenous Leukemia (CML) patients [2]. Gleevec is also effective for treating Gastrointestinal Stromal Tumors (GIST) because it blocks the hormone receptor Kit, which often causes GIST [2]. Despite these significant advances, the pathogenic mechanisms of many signal transducer proteins implicated in cancers remain unknown.

One interesting signal transducer protein that is a potential target for cancer therapeutic drugs is Vav1. Vav1 was identified as an oncogene using the nude mouse tumorigenicity assay [3]. In this assay, NIH3T3 cells co- transfected with DNA from several esophageal carcinomas and the pSV2neo plasmid (which carries the gene that confers resistance to the G418 drug) were injected into nude mice [3]. These experiments led to the isolation of a novel human oncogene, which was designated Vav, the sixth letter of the Hebrew alphabet, because it was the sixth oncogene detected in Dr. Barbacid's laboratory [3]. The isolation of the Vav oncogene led to the identification of its wild-type (wt) form [4, 5] and subsequent identification of two additional mammalian members of this protein family, Vav2 [6] and Vav3 [7]. Nucleotide sequence analysis of the first Vav oncogene isolated, now termed Vav1, revealed that it was activated in vitro by replacement of 67 residues of its amino-terminus with sequences of pSV2neo, co-transfected as a selectable marker [3-5]. Although initially identified as an oncogene [3], Vav1 has been subsequently acknowledged as an important signal transducer with a pivotal role in the hematopoietic system, where it is exclusively expressed [8-13], as will be detailed below.

This review will focus on our recent understanding of the involvement of Vav1 in human cancer, the mechanism of ectopic Vav1 expression in cancer and its 
mode of function. The newly identified mutations in Vav1 in human cancer will be discussed.

\section{Structure}

Vav1 contains many characteristic structural motifs important for its function as a versatile signal transducer (Figure 1) [8-11]. These include: 1) a calponin-homology domain ( $\mathrm{CH}$; amino acids 3-121) which, in other proteins, associates with F-actin [14]. The Vav1 CH domain does not associate with F-actin, but is critical for Vav1's involvement in calcium mobilization [15]; 2) an acidic motif (AC; amino acids 133-193) that contains three regulatory tyrosines (Y142; Y160 and Y174) [16]; 3) a DBL homology (DH) region (amino acids 199-373), which exhibits a guanine nucleotide exchange (GEF) activity towards the Rho family GTPases [17]; 4) a Pleckstrin homology domain (PH) (amino acids 404-505) that mediates interaction with phospholipids [18] resulting in Vav1 localization to the plasma membrane and regulation of Vav1 GEF activity [19]; 5) an atypical C1 (amino acids 515-564), which lacks the features required for lipid binding and instead might affect protein-protein interactions [20]; 6) a proline rich region (amino acids 606-610) that mediates binding of Vav proteins to Src homology 3 (SH3) containing proteins [21]; 7) a Src homology 2 (SH2) region (amino acids 672-746) that enables the binding of Vav1 to tyrosine phosphorylated proteins [22, 23]; 8) two SH3 domains (amino acids 615-659 and 786-841) that mediate interactions with proline-rich domains [22, 23]; and 9) two nuclear localization signals (NLS; amino acids 487-494 and 576-589) [24]. Finally, Vav1 contains multiple tyrosine residues that affect its activity $[25,26]$.

\section{Biological functions of Vav 1}

Vav1 participates in various cellular responses including actin cytoskeleton reorganization, gene transcription, and development and activation of immune cells. The role of Vav1 in the hematopoietic system has been extensively studied and reviewed [8-13] and therefore it will be only briefly summarized here.

The best-known function of Vav1 is its GEF activity for the Rho family of GTPases, an activity strictly dependent on tyrosine phosphorylation [7, 25, 27]. There have been conflicting reports on the substrate specificity of Vav1 [17, 27, 28], yet it is well accepted that Rac1 is the preferred substrate of Vav1 [17, 25, 27-29]. The nucleotide exchange activity of Vav1 on Cdc42, RhoA and RhoG is also enhanced, but to a lesser extent compared to Vav1's activity towards Rac [20].

In immune cells, endogenous Vav1 is tyrosine phosphorylated following activation of many receptors, including the T-cell receptor (TCR) $[22,23]$, B-cell receptor (BCR) [30], FcRI [31], cytokine receptors [32], NK receptors [33], chemokine receptors [34] and integrins [35]. The activation of Vav1 by these receptors leads to different outcomes depending on the specific hematopoietic cell type. For instance, Vav1 was shown to be associated with the formation of the immunological synapse (IS) in T cells [36, 37] and B cells [36] due to its activity as a regulator of cytoskeleton organization. Mice with Vav1-deficient $\mathrm{T}$ cells exhibited impaired cytoskeleton reorganization and impaired immune response [38, 39]. The GEF activity of Vav1 was also shown to be critical for activation of killing by Natural Killer (NK) cells [40]. In addition, $\mathrm{Vav1}^{-/}$mice are defective in their ability to eliminate tumors in vivo and in natural killing and antibody-dependent cellular cytotoxicity in vitro $[41,42]$. In macrophages, genetic

\section{$\nabla$ Missense mutation}

$\Delta$ Truncating mutation

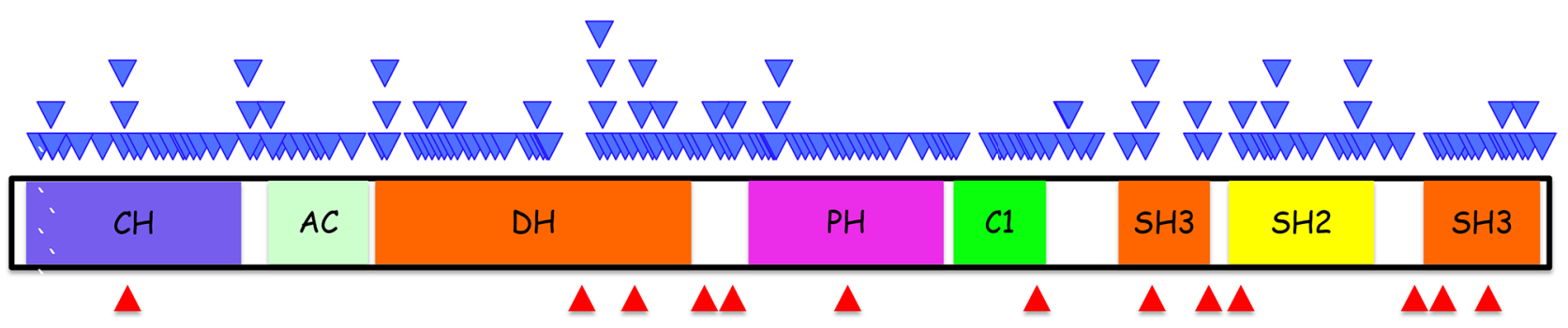

Figure 1: Schematic summary of Vav1 structure and location of various mutations identified in human cancers. Vav1 encodes the following domains: calponin-homology $(\mathrm{CH})$ domain; acidic (AC) motif, which contains 3 tyrosine residues; a DBL homology (DH) domain; a pleckstrin homology (PH) domain; a C1 domain; two SRC-homology 3 (SH3) domains; and a SRC-homology 2 (SH2) domain. The function of each region is detailed in the text. The location of missense mutations (light blue triangles) is indicated above the protein stricture and the location of truncations (red triangles) are depicted beneath. The information concerning these mutations is adapted from the catalogue of Somatic Mutations in Cancer (COSMIC) database. 
deletion of Vav1 shows that it is also required for Rac-dependent complement-mediated phagocytosis [43], cell migration [44] and macrophage chemotaxis to CSF-1 [45].

Vav1 also regulates the activity of multiple transcription factors in $\mathrm{T}$ cells in response to TCR stimulation, including Nuclear Factor of Activated T cells (NFAT), Activator Protein-1 (AP-1) and Nuclear Factor $\kappa \mathrm{B}(\mathrm{NF}-\kappa \mathrm{B})[15,46]$. Vav1's ability to trigger release of calcium from inner reservoirs in $T$ cells was found to be critical for this function [15].

Vav1 activates multiple signaling pathways, including the extracellular signal-regulated kinase (ERK) and c-Jun N-terminal kinase (JNK) pathways [25, 47, 48]. Murine Vav1-deficient T-cells exhibit defects in TCR-induced activation of ERK [15]. Moreover, Vav1 transduces TCR signals that lead to recruitment of the RasGEF, RasGRP1, Sos1 and Sos2 to LAT, leading to activation of ERK [49]. Also, over-expression of Vav1 and RasGRP1 in Jurkat T cells leads to hyperactivation of Ras [48].

Many of Vav1 functions are exerted via its ability to associate with other proteins [8-11]. For instance, several proteins associate with the $\mathrm{CH}$ region including Socs1, a downstream component of the Kit receptor tyrosine kinase signaling pathway [50]; ENX-1, a putative transcriptional regulator of homeobox gene expression [51]; Ly-GDI, a regulator of Rho GTPases [52]; and calmodulin [53]. The $\mathrm{SH} 2$ domain interacts with autophosphorylated tyrosine kinases such as ZAP-70 [54] and Syk [55] and with the adapter proteins: SLP76 [56] and Blnk [57]. Vav1's N-terminal SH3 domain binds the adapter protein Grb2 [58] shown to be necessary for translocation of Vav1 to the plasma membrane and its interaction with upstream tyrosine kinases in lymphoid cells [8]. The C-terminal $\mathrm{SH} 3$ domain of Vav1 forms complexes with a wide variety of proteins including cytoskeletal regulators (Zyxin) [59], RNA-binding proteins (hnRNP-K, hnRNP-C and Sam68) $[60,61]$, transcriptional modulators, ubiquitination factors, viral proteins, a Kruppel-like protein, and Dynamin 2 [8, $9,62]$. While the consequences of all these interactions are not yet known, Vav1's ability to interact with many proteins likely allows it to function in multiple signaling pathways, highlighting its involvement in multiple pathways.

Finally, Vav1 has an important role in hematopoietic cell development. $\mathrm{T}$ cells from Vav1-deficient mice demonstrated a partial block at the $\mathrm{CD}^{-} \mathrm{CD}^{-}$(double negative; $\mathrm{DN}$ ) to $\mathrm{CD}^{+} \mathrm{CD}^{+}$(double positive; DP) transition and also at the transition from $\mathrm{DP}$ to $\mathrm{CD} 4^{+} \mathrm{CD} 8^{-}$or $\mathrm{CD}^{-} \mathrm{CD}^{+}$single positive (SP) [63]. Experiments performed with mice lacking Vav1 and the other members of the Vav protein family, i.e., Vav2 and Vav3, led to a 100-fold reduction in the number of DP and SP thymocytes and peripheral $\mathrm{T}$ cells, indicating that Vav2 and Vav3 proteins partially compensate Vav1 function in thymocyte development [64].
In summary, we know that Vav1 is a versatile signal transducer, critical for numerous biological activities in the hematopietic system, largely through its Rho-GEF activity. However, its interaction with numerous and diverse effectors suggests it plays additional roles in various signaling cascades.

\section{VAV1 REGULATION}

Vav1 GEF activity is controlled through autoinhibition of the $\mathrm{DH}$ region, which is conferred by a $\alpha$-helix in the AC region. Tyr174 lies within this $\alpha$-helix and directly binds the GTPase interaction pocket of the DH domain, blocking access to its substrate and inhibiting Vav1 GEF activity. Phosphorylation releases Tyr174 from the binding pocket, relieving the auto-inhibition [28, 65]. It has been suggested that the Vav1 $\mathrm{CH}$ domain can bind to the $\mathrm{C} 1$ region, also occluding the $\mathrm{DH}$ domain and blocking access to Rac/RhoGTPases. The $\mathrm{CH}-\mathrm{C} 1$ interaction seemingly stabilizes the inhibitory Tyr174$\mathrm{DH}$ interaction. Indeed, deletion of this domain results in constitutively active GEF activity [66]. In addition, the $\mathrm{PH}$ domain has been shown to regulate Vav1 catalytic activity by interaction with two lipid products of PI3K: phosphatidylinositol 4, 5-biphosphosphate (PIP2) and phosphatidylinositol 3, 4, 5-triphosphosphate (PIP3) [19, 67]. Whereas binding of PIP3 moderately enhances the in vitro GEF activity of Vav1, binding to PIP2 has an inhibitory effect. Recent high resolution X-ray structure of DH-PH-C1 domains suggested that $\mathrm{PH}$ and $\mathrm{C} 1$ domains contribute to GEF activity by stabilizing the $\mathrm{DH}$ domain structure and not through direct contacts with Rac/ RhoGTPases [20].

\section{Vav 1 expression in human cancers}

In the past decade numerous studies reported the unexpected expression of Vav1, which is usually found only in the hematopietic system, in a variety of human cancers. Ectopic Vav1 expression was first noted in the neuroblastoma SK-N-MC cell line [68]. The Vav1 protein in SK-N-MC exhibited the same molecular weight, phosphorylation state, and ability to bind to EGF receptor as wild-type Vav1 and had no mutations [68]. A subsequent screen of 42 primary human neuroblastoma tumors revealed that the majority $(76 \%)$ expressed Vav1, suggesting for the first time that ectopic expression of wild type Vav1 might contribute to human cancer [68].

Vav1 was also identified in more than $50 \%$ of 95 pancreatic ductal adenocarcinoma (PDA) tumor specimens examined [69]. Patients with Vav1-positive tumors had a worse prognosis for survival compared to patients with Vav1-negative tumors [69]. Sequence analysis of the Vav1 cDNA from pancreatic cancer cell lines and tumors confirmed that they express intact wild-type Vav1 [69]. In addition, aberrant expression of Vav1 was found in $42 \%$ of 78 lung cancer cell lines examined, in $46 \%$ of 57 human primary lung cancer 
specimens [70] and in breast [71], ovarian and prostate cancers [26]. Grassilli et al., demonstrated that Vav1, a cytoplasmic expressed protein, is often found in the nucleus of early invasive breast tumors [72]. The high amounts of nuclear Vav1 in these tumors are positively correlated with low incidence of relapse [72]. Moreover, it was demonstrated that when it is expressed in the nucleus it can modulate genes that are associated with the metastatic process [72]. Several melanoma cell lines also express wild-type Vav1, including the highly metastatic BLM cells, although the level of protein expression was low and it was localized in the cell periphery near the plasma membrane [73]. Finally, a large screen of medulloblastomas identified widespread expression of Vav1 in the majority of specimens analyzed and Vav1 was demonstrated to play a critical role in medulloblastoma tumor maintenance, with Vav1 abrogation markedly reducing medulloblastoma growth [74].

Intriguingly, and perhaps counter-intuitively, Vav1 does not appear to be significantly involved in hematological malignancies [75]; however, from work reported thus far, this does not seem to be the case. Prieto-Sanchez et al. [76] examined Vav1 protein levels and phosphorylation status in 118 unselected cases of hematologic neoplasms. They found that Vav1 was phosphorylated and overexpressed in 10 of 14 cases of B-CLL with 13q deletion, however no change in its level of expression was recoreded in any of the myeloproliferative neoplasms examined [76]. Bertagnolo et al. have demonstrated in a series of studies that Vav1 is required for the retinoic ATRA-induced differentiation of human promyelocytic leukemia cell lines to neutrophils as well as PMA-induced maturation of these same cell lines to monocytes/macrophages [77, 78]. However, there is no evidence as to whether Vav1 has a role in this specific tumor development.

Thus, the accumulating data clearly point to an important role of ectopically expressed wild-type Vav1 in human cancer [24].

\section{WHY IS VAV1 EXPRESSED IN CANCER?}

Neither the physiological nor pathological regulation of Vav1 expression is completely understood. One mechanism suggested to play a role in ectopic expression of Vav1 in cancer of non-hematopoietic origin is the methylation status of the Vav1 promoter. Bisulfite sequencing revealed that the Vav1 promoter was completely unmethylated in human lymphocytes, but methylated to various degrees in healthy tissues that do not normally express Vav1 [79]. FernandezZapico, et al., demonstrated that epigenetic changes in the Vav1 gene, but not gene amplification, contributed to its aberrant expression in pancreatic cancer cell lines [69]. These results are further substantiated by a recent report indicating that Vav1 was identified by cross-species epigenetics to play a critical role in maintenance of Sonic Hedgehog (SHH) subgroup medulloblastoma tumors (MBSHH) [74]. This study identified widespread hypomethylation of Vav1, leading to its elevated expression, as a conserved aberrant epigenetic event that characterizes the majority of MBSHH tumors and is associated with poor outcome in MBSHH patients. These findings establish Vav1 as an epigenetically regulated oncogene with a key role in MBSHH maintenance [74].

Another mechanism emerged from our studies aimed at identifying transcription factors that regulate Vav1 expression [79]. We demonstrated that mutations in putative transcription factor binding sites at the Vav1 promoter affect its transcription in cells of different histological origin [79]. Among these sites is a consensus site for c-Myb, a hematopoietic-specific transcription factor also found in Vav1-expressing lung cancer cell lines. Depletion of c-Myb using siRNA led to a dramatic reduction in Vav1 expression in these cells [79]. Consistent with this, co-transfection of c-Myb activated transcription of a Vav1 promoter-luciferase reporter gene construct in lung cancer cells devoid of Vav1 expression. Together, these results indicate that c-Myb is involved in Vav1 expression in lung cancer cells. The possibility that additional transcription factors play a role in Vav1 expression in cancer cells remains to be explored.

\section{ROLE OF VAV1 IN HUMAN CANCER}

Vav1 functions physiologically in numerous pathways, therefore it is somewhat difficult to attribute its multiple activities in cancer to a particular pathway. Nonetheless, the main role attributed to Vav1 in cancer is its activity as a GEF for Rho/RacGTPases [9]. The Rho/ RacGTPases function as molecular switches in a variety of signaling pathways following stimulation of cell surface receptors. Rho/RacGTPases regulate numerous cellular processes that become dysregulated in cancer, including cytoskeleton organization, gene transcription, cell proliferation, migration, growth and survival [13, 80]. It therefore seems reasonable that defects in Rho/ RacGTPase pathway regulation may be involved in the development of cancer $[13,81]$. Consistent with this, various GEFs have been implicated recently in cancer $[13,26]$, and activation of Vav1 GEF activity following tyrosine phosphorylation has been demonstrated in EGF and PDGF stimulated NIH3T3 fibroblasts expressing Vav1 $[22,23]$ as well as in cancer cells, including neuroblastoma [68], pancreatic cancer [69] and lung cancer [70]. It is also activated following stimulation of CSF1R in lung cancer cells [82]. The truncated Vav1 oncogene first identified as an oncogene exhibited constitutive activity as a GEF [25]. 
A mutation at tyrosine 174 , leading to enhanced GEF activity, results in increased transformation [83], further highlighting the importance of the GEF activity of Vav1 for transformation. Also, Fernandez-Zapico et al., demonstrated that, unlike wild-type Vav1, a GEF-defective Vav1 mutant cannot restore proliferation of pancreatic cancer cells depleted of Vav1 [69]. Interestingly, Vav1 expression was required for proliferation even in the presence of mutant K-Ras in pancreatic and lung cancer, demonstrating the critical role of Vav1 in tumor development [69, 70]. Razidlo et al. recently reported that Vav1 is required for Rac1-mediated formation of lamellipodia and subsequent migration of tumor cells [84]. In addition, Vav1 is a potent regulator of transendothelial migration of leukocytes, and also contributes to CXCL12induced MT1-MMP expression and invasion by melanoma cells [73]. Additionally, Razidlo et al., demonstrated that Vav1 is involved in invasion and migration through the formation of invadopodia and matrix degradation [85]. This process requires Vav1 activation of $\mathrm{Cdc} 42$, demonstrating that in pancreatic tumor cells, ectopically expressed Vav1 can signal through multiple pathways. This is consistent with a previous report that Vav1-induced oncogenic transformation requires multiple signaling pathways, including Rac1, Cdc42, and RhoA, as well as NF $\kappa$ B and JNK [86]. Together, the above findings imply that GEF activity is critical for Vav1's role in cancer cell migration and invasion and suggest that ectopically expressed Vav1 acts as an upstream activator of Rac1, RhoA and possibly Cdc42 signaling pathways in response to extracellular stimulation, leading to cytoskeleton changes and ultimately to increased cell motility.

Activation of Vav1 also stimulates MAPK signaling cascades, which may contribute to cancer by enhancing cell mitogenic properties. MAPK function is related to multiple biologic processes such as cell proliferation, differentiation, death, migration, invasion and inflammation [87]. It is well established that once MAPK is abnormally activated, cancer may occur [87]. Numerous studies have described Vav1's physiological role in ERK signaling in the immune system [8]. Since Rho GTPases were reported to control cytoskeleton organization and cellular activities, such as the JNK (c-Jun N-terminal kinase) and p38 MAPK (mitogen-activated protein kinase) cascades [88], it is conceivable that Vav1 also controls these pathways in cancer. Indeed, we recently demonstrated that ERK phosphorylation is dependent on Vav1 activation in lung cancer cells [82].

Our recent data suggest that Vav1 may also contribute to cancers by regulating growth factor expression. We found that lung cancer cells depleted of Vav1 exhibit significantly reduced levels of the hematopoietic growth factor CSF1, suggesting that Vav1 propagates an autocrine feed forward loop by upregulating expression of growth factors [82].
Transcriptome analysis demonstrated that Vav1 depletion results in a marked reduction in CSF-1 expression. The association between Vav1 expression and CSF1 was further supported by signal transduction experiments, pointing to the involvement of Vav1 in regulating the lung cancer secretome [82]. Blocking ERK phosphorylation led to a decrease in CSF1 transcription, suggesting a role for ERK, a downstream effector of Vav1, in CSF1 expression [82]. CSF1silenced cells exhibited reduced focus formation, proliferation abilities, and growth in NOD/SCID mice. CSF1-silenced H358 cells resulted in significantly smaller tumors, showing increased fibrosis and a decrease in tumor infiltrating macrophages. Finally, immunohistochemical analysis of primary human lung tumors revealed a positive correlation between Vav1 and CSF1 expression, which was associated with tumor grade [82]. Our results suggest a potential crosstalk between cancer cells and the microenvironment controlled by CSF1/Vav1 signaling pathways. This indicates that Vav1 might be involved in additional protumorigenic pathways in addition to its GEF activity. It is noteworthy that lung cancer cells depleted of Vav1 also showed a decrease in EGF [82] and TGF $\alpha$ [70], further highlighting the association between Vav1 expression in cancer cells and the expression of autocrine/paracrine growth factors.

The possibility that Vav1 can stimulate secretion of autocrine ligands was also suggested for the human mammary epithelial cell line MCF-10A, in which expression of a constitutively active form of Vav1 promoted migration and morphological changes [89]. This increased migration was dependent on Vav1 GEF activity, which stimulated the Rac1-Pak pathway, and also on secretion of an autocrine EGF receptor ligand. We previously reported that the secretion of osteopontin, a CD44 and integrin ligand known to be associated with invasion, progression and metastasis, is upregulated by oncogenic Vav1 in NIH3T3 cells [90]. These data support the existence of feed-forward loops in which Vav1 regulates secretion of autocrine ligands leading to receptor stimulation and subsequent increases in Vav1 activation. The expression and function of many other proteins appear to be affected by Vav1 [82], yet the exact contribution of such proteins for Vav1-dependent tumorigenicity has not been explored.

Vav1 might also contribute to transformation by influencing cell cycle progression and gene transcription. Indeed, as shown in pancreatic cancer cells, EGF stimulation leads to tyrosine phosphorylation of Vav1, followed by the activation of a Rac1/Pak $1 / \mathrm{NF}-\kappa \mathrm{B}$ signaling pathway resulting in an increase in cyclin D1 which leads to enhanced pancreatic tumor cell proliferation [69]. This recurring theme suggests that Vav1 might contribute to the progression of cancer by regulating 
secretion of autocrine ligands critical for tumorigenicity, as well as affecting the expression of other proteins critical for various cellular functions.

The ability of Vav1 to contribute to cancer development was recently also attributed to its expression in cells of the microenvironment. Garcia JL et al., demonstrated by immunohistochemical survey of 59 high-grade gliomas that Vav1 is found in non-tumoural astrocyte-like cells in peri-tumor or peri-vascular locations, but not in the glioblastoma cells [91]. Thus, in this case, expression of Vav1 is linked to synergistic signaling cross-talk between cancer cells and infiltrating cells, a phenomenon that could have a role in the neoplastic process in glioblastoma tumors [91].

In summary, several of Vav1's intracellular mechanisms can contribute to tumorigenicity, including activation of RhoGTPases, activation of cyclin D1 and $\mathrm{NF}-\kappa \mathrm{B}$, and protein-protein interactions. Further research is required to provide more definitive insight into mechanisms underlying Vav1's role in human cancer.

\section{MUTATIONS IN VAV1 IN HUMAN CANCER}

While evidence over the last decade substantiated Vav1 overexpression in human cancer, the question remained whether mutations in Vav1 contribute to human cancers $[68-71,73,74,76]$. Based on data recently obtained from human genome sequencing coordinated by the Wellcome Trust Sanger Institute, Vav1 appears to be mutated in $\sim 1 \%$ of human cancer of multiple tissue origins (Figure 1, http://cancer.sanger.ac.uk/cosmic/gene/ analysis? $\ln =$ Vav $1 \& \ln 1=$ CBL\&start $=1 \&$ end $=907 \&$ coord $\mathrm{s}=\mathrm{AA} \% 3 \mathrm{AAA} \& \mathrm{sn}=\& \mathrm{ss}=\& \mathrm{hn}=\& \mathrm{sh}=\& \mathrm{id}=5003 \#)$. Some cancers exhibit a higher occurrence of Vav1 mutations, such as those originating in the biliary tract $(5.17 \%)$, endometrium $(3.23 \%)$, large intestine $(4.35 \%)$, and skin $(6.13 \%)$, possibly attesting to the importance of the molecular lesions in Vav1 in certain tissues (Figure 2). Since the isolation of Vav1 numerous studies have attempted to decipher its structure/function by introducing mutations at different domains. Therefore, it is interesting to compare the mutations found in human cancer to those experimental mutations. The mutations identified in Vav1 in human cancer span all its cardinal domains. CH Region: Mutations are at residues that are outside the backbone of this domain and are conserved in more than $62 \%$ of the sequences [92]. Several of these mutations (L17V, E59K, E84D, L88F, E95K, and W117R) are at isoleucine, leucine, valine, phenylalanine and tryptophan residues frequently found in this region, suggesting these residues may be involved in transformation. DH Region: Human cancer-associated mutations occur in highly conserved

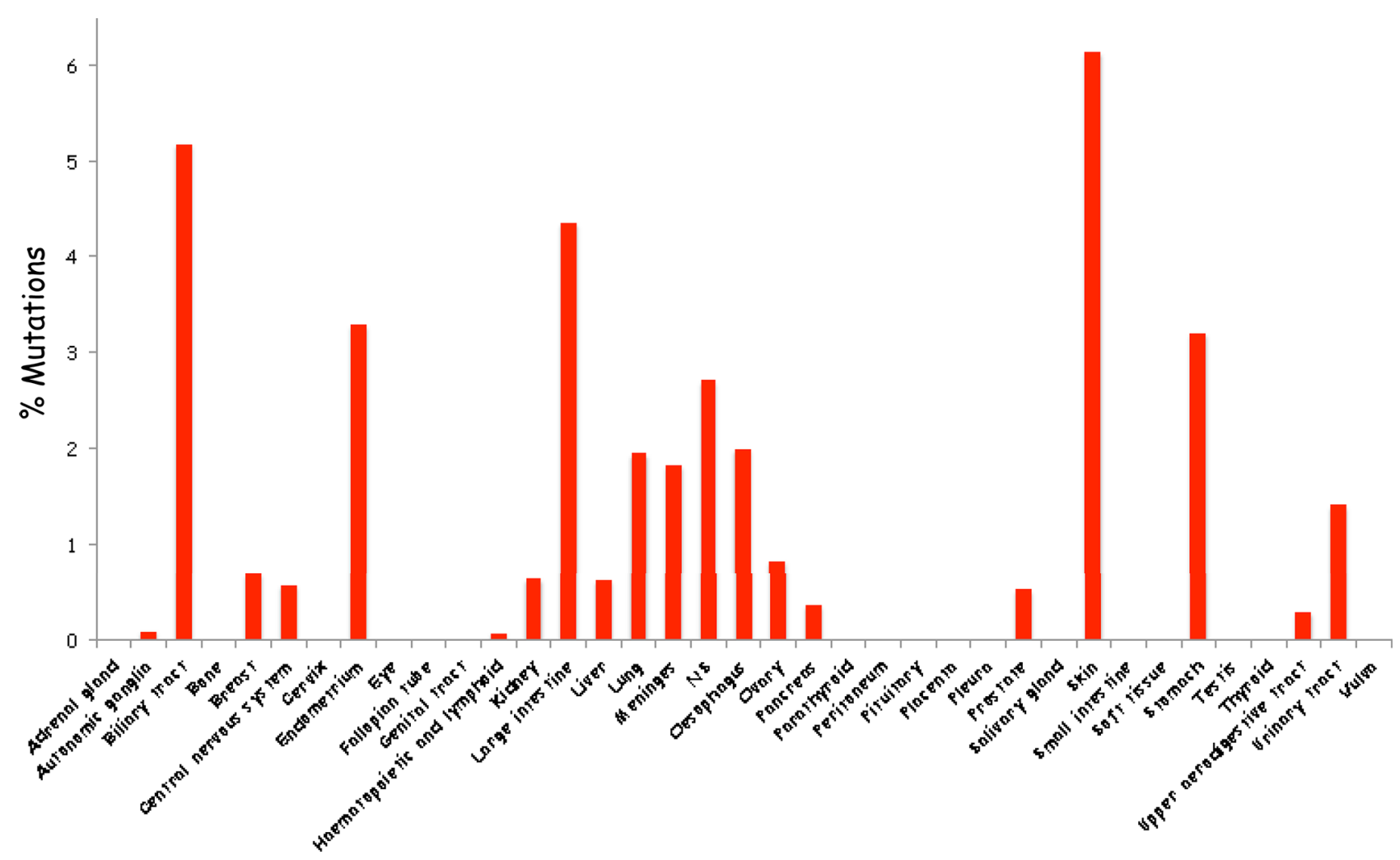

Figure 2: Schematic summary of Vav1 mutations in cancer of various tissue origins. The percentage of Vav1 mutations in numerous tissues was calculated according to the information available from the catalogue of Somatic Mutations in Cancer (COSMIC) database. 
residues, including E201K, L322M, D324Y, H337Y, L339I, T347M, V373F and E378K [93]. An additional mutant, $\mathrm{Y} 283 \mathrm{~F}$, is not conserved among all the proteins, but is shared with Dbl. PH Region: The only conserved residue within the $\mathrm{PH}$ region that has been shown to be mutated in human cancers is E408K [93]. C1 Region: Interestingly, several of the residues mutated in human cancers (Q542E, R548L, E556K and P562S), were previously mutated experimentally by Zugaza et al., [66] but with different amino-acid substitutions. Since Zugaza et al., substituted with alanine [66], the activity of the naturally occurring mutants may differ from Zuzaga's results. SH2 Domain. Several Vav1 mutations in cancer occur at highly conserved residues in the SH2 domain, including R678Q, E682E, G691R, and R696W.

For a long period it was believed that truncation of the amino-terminus was the only molecular lesion that converted wild-type Vav1 to a transforming gene in NIH3T3 fibroblasts $[4,5]$. The importance of the aminoterminus for Vav1 activity as a transforming gene was subsequently attributed to tyrosine 174 . Thus, mutation at tyrosine 174 greatly enhances the transforming activity of Vav1 [83], since it relieves the autoinhibition of GEF activity by the acidic region [28]. One additional mechanism for Vav1 activation was recently reported by Razanadrakoto et al., [94] demonstrating that a mutation at D797 (D797N) in the carboxy SH3 region of wild-type Vav1 endowed the protein with transforming properties [94]. The same mutation introduced in the Vav1 oncogene did not change its transforming potential, suggesting that they operate in a similar manner [95]. Although numerous mutations have been experimentally introduced in Vav1 throughout the years, including in the $\mathrm{DH}, \mathrm{PH}, \mathrm{C} 1$, both $\mathrm{SH} 3$ regions and the $\mathrm{SH} 2$, none led to increased transformation of Vav1-expressing NIH3T3 fibroblasts $[26,66,95,96]$, except the ones mentioned above. Therefore, it remains to be tested whether the cancer-identified Vav1 mutants are transforimg in such experimental conditions.

The pattern of mutations in Vav1 is puzzling. Both missense mutations and protein-truncating alterations are found throughout the Vav1 protein (Figure 1). Vogelstein and colleagues concluded that such a pattern of mutations is typical for tumor suppressor genes, while oncogenes are recurrently mutated at the same amino acid positions [97]. However, in view of the extreme functional complexity of the Vav1 protein, we cannot draw the same conclusion for Vav1 without further study. It is possible that aberrant function of each of the different domains of Vav1 can have different consequences in different cell types, or different pathophysiological processes, leading independently to transformation.

Several examples support the idea that genes can have dual roles as oncogenes and tumor suppressor genes depending on the specific mutation and tissue distribution. p53, a well-known tumor suppressor gene, can also function as an oncogene when it carries a gainof-function mutation [98]. Thus, some mutant p53 proteins gain oncogenic functions through which they actively contribute to establishment, maintenance and spreading of cancer cells [98]. Also, some functional studies suggest that NOTCH1 is an oncogene, whereas others suggest it is a tumor suppressor gene [99]. In hematological malignancies such as lymphomas and leukemias, NOTCH1 mutations were often recurrent and did not truncate the predicted protein [100], while in certain solid tumors, the mutations were not recurrent and were usually inactivating [101]. Thus different mutations to the same protein (NOTCH1) lead to its involvement in different tumor types through distinct mechanisms.

We recently demonstrated that Vav1 plays a dual role as a pro- or an anti-apoptotic protein in breast cancer cells, depending on whether the cells express p53 [71]. p53 is required for the pro-apoptotic effect of Vav1 in these breast cancer cell lines [71]. Whether these experiments point to a possible dual role of Vav1 in cancer, depending on the specific mutation and the specific cell-type, remains to be carefully studied. In light of the fact that Vav1 is mutated in just 1\% of 20427 cancer specimen analyzed, and in view of its complex biochemical structure and diverse cellular functions, it is prudent to await the identification of a larger number of mutations before we draw conclusions about the true identity of Vav1 mutants (oncogene/tumor suppressor gene), as suggested by Lawrence et al., who explored the feasibility of creating a comprehensive catalogue of cancer genes [102].

\section{ROLE OF VAV2 AND VAV3 IN HUMAN CANCER}

Whereas the expression of Vav1 appears to be predominantly limited to the hematopoietic system, Vav2 and Vav3 are expressed more ubiquitously [3, 6, 7]. The various members of the Vav family of proteins (Vav1, Vav2 and Vav3) exhibit redundant as well as distinct functions in development $[64,103]$. Both Vav2 and Vav3 have been implicated in cancer. High expression of Vav2 is implicated in cancers such as oral squamous cell carcinoma [104], squamous carcinomas of the head and neck [105], and prostate cancer [106]. Also, high levels of Vav3 have been observed in various types of cancers, including glioblastoma [107], prostate cancer [108] and colorectal cancer [109]. Vav3 was also shown to be significantly upregulated in breast cancers compared with benign breast diseases [110, 111]. Furthermore, Vav3 was identified as a biomarker of a poor prognosis in breast and ovarian cancers [112, 113]. Like Vav1, Vav2 and Vav3 become oncogenic following $\mathrm{N}$-terminal truncation [7, 27], yet there appears to be Vav isoform-distinct functions in cancer. For instance, specific depletion of only Vav1 in pancreatic cancer cell lines led to inhibition of their growth, despite the continuous expression 
of Vav2 [69]. Moreover, knockdown of Vav2 in these cells did not alter their growth [69], suggesting that Vav1 and Vav2 play different roles in pancreatic cancer cells. Contrary, depletion of Vav2 and Vav3 could result in a dramatic effect on tumor growth of a different histological origin. Thus, reduction of Vav2 and Vav3 expression in mouse mammary tumor cells led to a decline in metastatic growth, similar to the effect of Vav1 depletion in pancreatic cancer cells [114]. These results further highlight the fact that the various members of the Vav family of proteins, Vav1, Vav2 and Vav3, may have different roles in human cancer.

\section{CONCLUDING REMARKS}

Vav1 is a signal transducer protein that functions exclusively in the hematopoietic system under normal physiological conditions. It participates in signal transduction events through tyrosine phosphorylationdependent guanine nucleotide exchange activity. While Vav1 was first identified as an oncogene capable of inducing transformation in NIH3T3 fibroblasts, accumulating results from the past decade clearly indicate its participation in human cancer through ectopic overexpression. Research on Vav1 in recent years has cycled between furthering our understanding of its physiological function in the hematopoietic system and studying its involvement in human malignancies. While it is now clear that Vav1 expression is deregulated in some cancers, leading to expression outside the hematopoietic system, it also emerges now as a mutated gene in human cancers of various origins; however, the activity and contribution of the various mutations is still unclear. The biological importance of the Vav1 mutants identified in human cancer needs further exploration, including testing the role of the various mutants in cognate tissues, assessing GEF activity of mutants, and testing their ability to associate with other proteins. Despite these many open questions, the existing data suggest Vav1 as a promising target for drug design, especially blocking its GEF activity, as was recently implied by Razidlo et al., who demonstrated that inhibition of Vav1 by drugs leads to inhibition of pancreatic cancer metastasis [115].

\section{ACKNOWLEDGMENTS}

I am indebted to Dr. Susan Lewis for editing the manuscript and to Prof. Eli Pikarsky for his critical advice.

\section{FUNDING INFORMATION}

This work was partially supported by grants from the Israel Academy of Sciences; Israel Cancer Research Foundation; the Israeli Cancer Association (ICA), with the generous assistance of the London friends of ICA in memory of the late Haim Yacobi; The Hubert H. Humphrey Center for Experimental Medicine and Cancer Research; The Alex U. Soyka Pancreatic Cancer Research Program and a donation from Vibeke Lichten.

\section{CONFLICTS OF INTEREST}

None declared.

\section{REFERENCES}

1. Sjoblom T, Jones S, Wood LD, Parsons DW, Lin J, Barber TD, Mandelker D, Leary RJ, Ptak J, Silliman N, Szabo S, Buckhaults P, Farrell C, et al. The consensus coding sequences of human breast and colorectal cancers. Science. 2006; 314:268-274.

2. Shawver LK, Slamon D, Ullrich A. Smart drugs: tyrosine kinase inhibitors in cancer therapy. Cancer Cell. 2002; $1: 117-123$.

3. Katzav S, Martin-Zanca D, Barbacid M. Vav, a novel human oncogene derived from a locus ubiquitously expressed in hematopoietic cells. EMBO J. 1989; 8:2283-2290.

4. Coppola J, Bryant S, Koda T, Conway D, Barbacid M. Mechanism of activation of the Vav protooncogene. Cell Growth Differ. 1991; 2:95-105.

5. Katzav S, Cleveland JL, Heslop HE, Pulido D. Loss of the amino-terminal helix-loop-helix domain of the Vav protooncogene activates its transforming potential. Mol Cell Biol. 1991; 11:1912-1920.

6. Henske EP, Jozwiak S, Bovey CM, Ramlakhan S, Haines JL, Kwiatkowski DJ. Identification of VAV2 on $9 \mathrm{q} 34$ and its exclusion as the tuberous sclerosis gene TSC1. Ann Hum Genet. 1995; 59:25-37.

7. Movilla N, Bustelo XR. Biological and regulatory properties of Vav-3, a new member of the Vav family of oncoproteins. Mol Cell Biol. 1999; 19:7870-7885.

8. Tybulewicz VL. Vav-family proteins in T-cell signalling. Curr Opin Immunol. 2005; 17:267-274.

9. Bustelo XR. Regulatory and signaling properties of the Vav family. Mol Cell Biol. 2000; 20:1461-1477.

10. Katzav S. Vav1: an oncogene that regulates specific transcriptional activation of T cells. Blood. 2004; 103:2443-2451.

11. Katzav S. Vav1: a hematopoietic signal transduction molecule involved in human malignancies. Int J Biochem Cell Biol. 2009; 41:1245-1248.

12. Kurosaki T. Regulation of BCR signaling. Mol Immunol. 2011; 48:1287-1291.

13. Cook DR, Rossman KL, Der CJ. Rho guanine nucleotide exchange factors: regulators of Rho GTPase activity in development and disease. Oncogene. 2014; 33:4021-4035. 
14. Castresana J, Saraste M. Does Vav Bind to F-Actin through a Ch Domain. Febs Letters. 1995; 374:149-151.

15. Costello PS, Walters AE, Mee PJ, Turner M, Reynolds LF, Prisco A, Sarner N, Zamoyska R, Tybulewicz VL. The Rho-family GTP exchange factor Vav is a critical transducer of $\mathrm{T}$ cell receptor signals to the calcium, ERK, and NF-kappaB pathways. Proc Natl Acad Sci U S A. 1999; 96:3035-3040.

16. Abe K, Whitehead IP, O'Bryan JP, Der CJ. Involvement of $\mathrm{NH}(2)$-terminal sequences in the negative regulation of Vav signaling and transforming activity. J Biol Chem. 1999; 274:30410-30418.

17. Movilla N, Dosil M, Zheng Y, Bustelo XR. How Vav proteins discriminate the GTPases Rac1 and RhoA from Cdc42. Oncogene. 2001; 20:8057-8065.

18. Wang DS, Shaw G. The association of the $\mathrm{C}$-terminal region of beta I sigma II spectrin to brain membranes is mediated by a $\mathrm{PH}$ domain, does not require membrane proteins, and coincides with a inositol-1,4,5 triphosphate binding site. Biochem Biophys Res Commun. 1995; 217:608-615.

19. Das B, Shu X, Day GJ, Han J, Krishna UM, Falck JR, Broek D. Control of intramolecular interactions between the pleckstrin homology and Dbl homology domains of Vav and Sos1 regulates Rac binding. J Biol Chem. 2000; 275:15074-15081.

20. Rapley J, Tybulewicz VL, Rittinger K. Crucial structural role for the $\mathrm{PH}$ and $\mathrm{C} 1$ domains of the Vav1 exchange factor. EMBO Rep. 2008; 9:655-661.

21. Ramos-Morales F, Romero F, Schweighoffer F, Bismuth G, Camonis J, Tortolero M, Fischer S. The proline-rich region of Vav binds to Grb2 and Grb3-3. Oncogene. 1995; 11:1665-1669.

22. Margolis B, Hu P, Katzav S, Li W, Oliver JM, Ullrich A, Weiss A, Schlessinger J. Tyrosine phosphorylation of Vav proto-oncogene product containing $\mathrm{SH} 2$ domain and transcription factor motifs. Nature. 1992; 356:71-74.

23. Bustelo XR, Ledbetter JA, Barbacid M. Product of Vav proto-oncogene defines a new class of tyrosine protein kinase substrates. Nature. 1992; 356:68-71.

24. Katzav S. Flesh and blood: the story of Vav1, a gene that signals in hematopoietic cells but can be transforming in human malignancies. Cancer Lett. 2007; 255:241-254.

25. Crespo P, Schuebel KE, Ostrom AA, Gutkind JS, Bustelo XR. Phosphotyrosine-dependent activation of Rac-1 GDP/GTP exchange by the Vav proto-oncogene product. Nature. 1997; 385:169-172.

26. Lazer G, Katzav S. Guanine nucleotide exchange factors for RhoGTPases: Good therapeutic targets for cancer therapy? Cellular Signalling. 2011; 23:969-979.

27. Schuebel KE, Movilla N, Rosa JL, Bustelo XR. Phosphorylation-dependent and constitutive activation of Rho proteins by wild-type and oncogenic Vav-2. EMBO J. 1998; 17:6608-6621.
28. Aghazadeh B, Lowry WE, Huang XY, Rosen MK. Structural basis for relief of autoinhibition of the Dbl homology domain of proto-oncogene Vav by tyrosine phosphorylation. Cell. 2000; 102:625-633.

29. Han J, Das B, Wei W, Van Aelst L, Mosteller RD, Khosravi-Far R, Westwick JK, Der CJ, Broek D. Lck regulates Vav activation of members of the Rho family of GTPases. Mol Cell Biol. 1997; 17:1346-1353.

30. Bustelo XR, Barbacid M. Tyrosine phosphorylation of the Vav proto-oncogene product in activated B cells. Science. 1992; 256:1196-1199.

31. Manetz TS, Gonzalez-Espinosa C, Arudchandran R, Xirasagar S, Tybulewicz V, Rivera J. Vav1 regulates phospholipase cgamma activation and calcium responses in mast cells. Mol Cell Biol. 2001; 21:3763-3774.

32. Evans GA, Howard OM, Erwin R, Farrar WL. Interleukin-2 induces tyrosine phosphorylation of the Vav proto-oncogene product in human T cells: lack of requirement for the tyrosine kinase lck. Biochem J. 1993; 294:339-342.

33. Galandrini R, Palmieri G, Piccoli M, Frati L, Santoni A. Role for the Rac1 exchange factor Vav in the signaling pathways leading to NK cell cytotoxicity. J Immunol. 1999; 162:3148-3152.

34. Garcia-Bernal D, Wright N, Sotillo-Mallo E, NombelaArrieta C, Stein JV, Bustelo XR, Teixidó J. Vav1 and Rac control chemokine-promoted $\mathrm{T}$ lymphocyte adhesion mediated by the integrin alpha4beta1. Mol Biol Cell. 2005; 16:3223-3235.

35. Zheng L, Sjolander A, Eckerdal J, Andersson T. Antibodyinduced engagement of beta 2 integrins on adherent human neutrophils triggers activation of p21 ras through tyrosine phosphorylation of the protooncogene product Vav. Proc Natl Acad Sci U S A. 1996; 93:8431-8436.

36. Arana E, Vehlow A, Harwood NE, Vigorito E, Henderson R, Turner M, Tybulewicz VL, Batista FD. Activation of the small GTPase Rac2 via the B cell receptor regulates $\mathrm{B}$ cell adhesion and immunological-synapse formation. Immunity. 2008; 28:88-99.

37. Krawczyk C, Oliveira-dos-Santos A, Sasaki T, Griffiths E, Ohashi PS, Snapper S, Alt F, Penninger JM. Vav1 controls integrin clustering and $\mathrm{MHC} /$ peptide-specific cell adhesion to antigen-presenting cells. Immunity. 2002; 16:331-343.

38. Fischer KD, Kong YY, Nishina H, Tedford K, Marengère LE, Kozieradzki I, Sasaki T, Starr M, Chan G, Gardener S, Nghiem MP, Bouchard D, Barbacid M, et al. $\mathrm{Vav}$ is a regulator of cytoskeletal reorganization mediated by the T-cell receptor. Curr Biol. 1998; 8:554-562.

39. Holsinger LJ, Graef IE, Swat W, Chi T, Bautista DM, Davidson L, Lewis RS, Alt FW, Crabtree GR. Defects in actin-cap formation in Vav-deficient mice implicate an actin requirement for lymphocyte signal transduction. Curr Biol. 1998; 8:563-572. 
40. Billadeau DD, Brumbaugh KM, Dick CJ, Schoon RA, Bustelo XR, Leibson PJ. The Vav-Rac1 pathway in cytotoxic lymphocytes regulates the generation of cell-mediated killing. J Exp Med. 1998; 188:549-559.

41. Chan G, Hanke T, Fischer KD. Vav-1 regulates NK T cell development and NK cell cytotoxicity. Eur J Immunol. 2001; 31:2403-2410.

42. Colucci F, Rosmaraki E, Bregenholt S, Samson SI, Di Bartolo V, Turner M, Vanes L, Tybulewicz V, Di Santo JP. Functional dichotomy in natural killer cell signaling: Vav1-dependent and -independent mechanisms. J Exp Med. 2001; 193:1413-1424.

43. Hall AB, Gakidis MA, Glogauer M, Wilsbacher JL, Gao S, Swat W, Brugge JS. Requirements for Vav guanine nucleotide exchange factors and Rho GTPases in FcgammaRand complement-mediated phagocytosis. Immunity. 2006; 24:305-316.

44. Wells CM, Bhavsar PJ, Evans IR, Vigorito E, Turner M, Tybulewicz V, Ridley AJ. Vav1 and Vav2 play different roles in macrophage migration and cytoskeletal organization. Exp Cell Res. 2005; 310:303-310.

45. Vedham V, Phee H, Coggeshall KM. Vav activation and function as a rac guanine nucleotide exchange factor in macrophage colony-stimulating factor-induced macrophage chemotaxis. Mol Cell Biol. 2005; 25:4211-4220.

46. Wu J, Katzav S, Weiss A. A functional T-cell receptor signaling pathway is required for p95Vav activity. Mol Cell Biol. 1995; 15:4337-4346.

47. Kaminuma O, Deckert M, Elly C, Liu YC, Altman A. VavRac1-mediated activation of the c-Jun N-terminal kinase/cJun/AP-1 pathway plays a major role in stimulation of the distal NFAT site in the interleukin-2 gene promoter. Mol Cell Biol. 2001; 21:3126-3136.

48. Villalba M, Coudronniere N, Deckert M, Teixeiro E, Mas P, Altman A. A novel functional interaction between $\mathrm{Vav}$ and PKCtheta is required for TCR-induced T cell activation. Immunity. 2000; 12:151-160.

49. Reynolds LF, de Bettignies C, Norton T, Beeser A, Chernoff J, Tybulewicz VL. Vav1 transduces T cell receptor signals to the activation of the Ras/ERK pathway via LAT, Sos, and RasGRP1. J Biol Chem. 2004; 279:18239-18246.

50. De Sepulveda P, Ilangumaran S, Rottapel R. Suppressor of cytokine signaling-1 inhibits VAV function through protein degradation. J Biol Chem. 2000; 275:14005-14008.

51. Hobert $\mathrm{O}$, Jallal $\mathrm{B}$, Ullrich A. Interaction of Vav with ENX-1, a putative transcriptional regulator of homeobox gene expression. Mol Cell Biol. 1996; 16:3066-3073.

52. Groysman M, LIftshitz C, Russek N, Katzav S. Vav, a GDP/GTP nucleotide exchange factor, interacts with GDIs, proteins that inhibit GDP/GTP dissociation. FEBS Lett. 2000; 467:75-80.

53. Zhou Z, Yin J, Dou Z, Tang J, Zhang C, Cao Y. The calponin homology domain of Vav1 associates with calmodulin and is prerequisite to $\mathrm{T}$ cell antigen receptorinduced calcium release in Jurkat $\mathrm{T}$ lymphocytes. J Biol Chem. 2007; 282:23737-23744.

54. Katzav S, Sutherland M, Packham G, Yi T, Weiss A. The protein tyrosine kinase ZAP-70 can associate with the SH2 domain of proto-Vav. J Biol Chem. 1994; 269:32579-32585.

55. Darby C, Geahlen RL, Schreiber AD. Stimulation of macrophage Fc gamma RIIIA activates the receptor-associated protein tyrosine kinase Syk and induces phosphorylation of multiple proteins including p95Vav and p62/GAPassociated protein. J Immunol. 1994; 152:5429-5437.

56. Wu J, Motto DG, Koretzky GA, Weiss A. Vav and SLP-76 interact and functionally cooperate in IL-2 gene activation. Immunity. 1996; 4:593-602.

57. Johmura S, Oh-hora M, Inabe K, Nishikawa Y, Hayashi K, Vigorito E, Kitamura D, Turner M, Shingu K, Hikida M, Kurosaki T. Regulation of Vav localization in membrane rafts by adaptor molecules Grb2 and BLNK. Immunity. 2003; 18:777-787.

58. Ye ZS, Baltimore D. Binding of Vav to Grb2 through dimerization of Src homology 3 domains. Proc Natl Acad Sci U S A. 1994; 91:12629-12633.

59. Hobert O, Schilling JW, Beckerle MC, Ullrich A, Jallal B. SH3 domain-dependent interaction of the proto-oncogene product Vav with the focal contact protein zyxin. Oncogene. 1996; 12:1577-1581.

60. Hobert O, Jallal B, Schlessinger J, Ullrich A. Novel signaling pathway suggested by $\mathrm{SH} 3$ domain-mediated p95Vav/ heterogeneous ribonucleoprotein $\mathrm{K}$ interaction. J Biol Chem. 1994; 269:20225-20228.

61. Lazer G, Pe'er L, Schapira V, Richard S, Katzav S. The association of Sam68 with Vav1 contributes to tumorigenesis. Cell Signal. 2007; 19:2479-2486.

62. Turner M, Billadeau DD. VAV proteins as signal integrators for multi-subunit immune-recognition receptors. Nat Rev Immunol. 2002; 2:476-486.

63. Turner M, Mee PJ, Walters AE, Quinn ME, Mellor AL, Zamoyska R, Tybulewicz VL. A requirement for the Rhofamily GTP exchange factor Vav in positive and negative selection of thymocytes. Immunity. 1997; 7:451-460.

64. Fujikawa K, Miletic AV, Alt FW, Faccio R, Brown T, Hoog J, Fredericks J, Nishi S, Mildiner S, Moores SL, Brugge J, Rosen FS, Swat W. Vav1/2/3-null mice define an essential role for Vav family proteins in lymphocyte development and activation but a differential requirement in MAPK signaling in T and B cells. J Exp Med. 2003; 198:1595-1608.

65. Yu B, Martins IR, Li P, Amarasinghe GK, Umetani J, Fernandez-Zapico ME, Billadeau DD, Machius M, Tomchick DR, Rosen MK. Structural and energetic mechanisms of cooperative autoinhibition and activation of Vav1. Cell. 2010; 140:246-256. 
66. Zugaza JL, Lopez-Lago MA, Caloca MJ, Dosil M, Movilla N, Bustelo XR. Structural determinants for the biological activity of Vav proteins. J Biol Chem. 2002; 277:45377-45392.

67. Han J, Luby-Phelps K, Das B, Shu X, Xia Y, Mosteller RD, Krishna UM, Falck JR, White MA, Broek D. Role of substrates and products of PI 3-kinase in regulating activation of Rac-related guanosine triphosphatases by Vav. Science. 1998; 279:558-560.

68. Hornstein I, Pikarsky E, Groysman M, Amir G, PeylanRamu N, Katzav S. The haematopoietic specific signal transducer Vav1 is expressed in a subset of human neuroblastomas. J Pathol. 2003; 199:526-533.

69. Fernandez-Zapico ME, Gonzalez-Paz NC, Weiss E, Savoy DN, Molina JR, Fonseca R, Smyrk TC, Chari ST, Urrutia R, Billadeau DD. Ectopic expression of VAV1 reveals an unexpected role in pancreatic cancer tumorigenesis. Cancer Cell. 2005; 7:39-49.

70. Lazer G, Idelchuk Y, Schapira V, Pikarsky E, Katzav S. The haematopoietic specific signal transducer Vav1 is aberrantly expressed in lung cancer and plays a role in tumourigenesis. J Pathol. 2009; 219:25-34.

71. Sebban S, Farago M, Gashai D, Ilan L, Pikarsky E, BenPorath I, Katzav S. Vav1 fine tunes p53 control of apoptosis versus proliferation in breast cancer. PLoS One. 2013; 8:e54321.

72. Grassilli S, Brugnoli F, Lattanzio R, Rossi C, Perracchio L, Mottolese M, Marchisio M, Palomba M, Nika E, Natali PG, Piantelli M, Capitani S, Bertagnolo V. High nuclear level of Vav1 is a positive prognostic factor in early invasive breast tumors: a role in modulating genes related to the efficiency of metastatic process. Oncotarget. 2014; 5:4320-4336.

73. Bartolome RA, Molina-Ortiz I, Samaniego R, SanchezMateos P, Bustelo XR, Teixidó J. Activation of Vav/Rho GTPase signaling by CXCL12 controls membrane-type matrix metalloproteinase-dependent melanoma cell invasion. Cancer Res. 2006; 66:248-258.

74. Lindsey JC, Kawauchi D, Schwalbe EC, Solecki DJ, Selby MP, McKinnon PJ, Olson JM, Hayden JT, Grundy RG, Ellison DW, Williamson D, Bailey S, Roussel MF, Clifford SC. Cross-species epigenetics identifies a critical role for VAV1 in SHH subgroup medulloblastoma maintenance. Oncogene. 2014.

75. Oberley MJ, Wang DS, Yang DT. Vav1 in hematologic neoplasms. Am J Blood Res. 2012; 2:1-8.

76. Prieto-Sanchez RM, Hernandez JA, Garcia JL, Gutierrez NC, San Miguel J, Bustelo XR, Hernández JM. Overexpression of the VAV proto-oncogene product is associated with B-cell chronic lymphocytic leukaemia displaying loss on 13q. Br J Haematol. 2006; 133:642-645.

77. Bertagnolo V, Brugnoli F, Grassilli S, Nika E, Capitani S. Vav1 in differentiation of tumoral promyelocytes. Cell Signal. 2012; 24:612-620.
78. Bertagnolo V, Grassilli S, Bavelloni A, Brugnoli F, Piazzi M, Candiano G, Petretto A, Benedusi M, Capitani S. Vav1 modulates protein expression during ATRA-induced maturation of APL-derived promyelocytes: a proteomicbased analysis. J Proteome Res. 2008; 7:3729-3736.

79. Ilan L, Katzav S. Human Vav1 expression in hematopoietic and cancer cell lines is regulated by c-Myb and by $\mathrm{CpG}$ methylation. PLoS One. 2012; 7:e29939.

80. Bouzahzah B, Albanese C, Ahmed F, Pixley F, Lisanti MP, Segall JD, Condeelis J, Joyce D, Minden A, Der CJ, Chan A, Symons M, Pestell RG. Rho family GTPases regulate mammary epithelium cell growth and metastasis through distinguishable pathways. Mol Med. 2001; 7:816-830.

81. Lin R, Bagrodia S, Cerione R, Manor D. A novel Cdc42Hs mutant induces cellular transformation. Curr Biol. 1997; 7:794-797.

82. Sebban S, Farago M, Rabinovich S, Lazer G, Idelchuck Y, Ilan L, Pikarsky E, Katzav S. Vav1 promotes lung cancer growth by instigating tumor-microenvironment cross-talk via growth factor secretion. Oncotarget. 2014; 5:9214-9226.

83. Lopez-Lago M, Lee H, Cruz C, Movilla M, Bustelo XR. Tyrosine phosphorylation mediates both activation and downmodulation of the biological activity of Vav. Mol Cell Biol. 2000; 20:1678-1691.

84. Razidlo GL, Wang Y, Chen J, Krueger EW, Billadeau DD, McNiven MA. Dynamin 2 potentiates invasive migration of pancreatic tumor cells through stabilization of the Rac1 GEF Vav1. Dev Cell. 2013; 24:573-585.

85. Razidlo GL, Schroeder B, Chen J, Billadeau DD, McNiven MA. Vav1 as a central regulator of invadopodia assembly. Curr Biol. 2014; 24:86-93.

86. Palmby TR, Abe K, Karnoub AE, Der CJ. Vav transformation requires activation of multiple GTPases and regulation of gene expression. Mol Cancer Res. 2004; 2:702-711.

87. Lei YY, Wang WJ, Mei JH, Wang CL. Mitogen-activated protein kinase signal transduction in solid tumors. Asian Pac J Cancer Prev. 2014; 15:8539-8548.

88. Hall A. Rho GTPases and the control of cell behaviour. Biochem Soc Trans. 2005; 33:891-895.

89. Wilsbacher JL, Moores SL, Brugge JS. An active form of Vav1 induces migration of mammary epithelial cells by stimulating secretion of an epidermal growth factor receptor ligand. Cell Commun Signal. 2006; 4:5.

90. Schapira V, Lazer G, Katzav S. Osteopontin is an oncogenic Vav1- but not wild-type Vav1-responsive gene: implications for fibroblast transformation. Cancer Res. 2006; 66:6183-6191.

91. Garcia JL, Couceiro J, Gomez-Moreta JA, Gonzalez Valero JM, Briz AS, Sauzeau V, Lumbreras E, Delgado M, Robledo C, Almunia ML, Bustelo XR, Hernandez JM. Expression of VAV1 in the tumour microenvironment of glioblastoma multiforme. J Neurooncol. 2012; 110:69-77. 
92. Banuelos S, Saraste M, Djinovic Carugo K. Structural comparisons of calponin homology domains: implications for actin binding. Structure. 1998; 6:1419-1431.

93. Habets GG, Scholtes EH, Zuydgeest D, van der Kammen RA, Stam JC, Berns A, Collard JG. Identification of an invasion-inducing gene, Tiam-1, that encodes a protein with homology to GDP-GTP exchangers for Rho-like proteins. Cell. 1994; 77:537-549.

94. Razanadrakoto L, Cormier F, Lauriente V, Dondi E, Gardano L, Katzav S, Guittat L, Varin-Blank N. Mutation of Vav1 adaptor region reveals a new oncogenic activation. Oncotarget. 2015; 6:2524-2537.

95. Groysman M, Nagano M, Shaanan B, Katzav S. Mutagenic analysis of Vav reveals that an intact SH3 domain is required for transformation. Oncogene. 1998; 17:1597-1606.

96. Katzav S. Single point mutations in the SH2 domain impair the transforming potential of Vav and fail to activate proto-Vav. Oncogene. 1993; 8:1757-1763.

97. Vogelstein B, Papadopoulos N, Velculescu VE, Zhou S, Diaz Jr. LA, Kinzler KW. Cancer genome landscapes. Science. 2013; 339:1546-1558.

98. Santoro R, Strano S, Blandino G. Transcriptional regulation by mutant p53 and oncogenesis. Subcell Biochem. 2014; 85:91-103.

99. Nicolas M, Wolfer A, Raj K, Kummer JA, Mill P, van Noort M, Hui CC, Clevers H, Dotto GP, Radtke F. Notch1 functions as a tumor suppressor in mouse skin. Nat Genet. 2003; 33:416-421.

100. Weng AP, Ferrando AA, Lee W, Morris JP 4th, Silverman LB, Sanchez-Irizarry C, Blacklow SC, Look AT, Aster JC. Activating mutations of NOTCH1 in human $\mathrm{T}$ cell acute lymphoblastic leukemia. Science. 2004; 306:269-271.

101. Agrawal N, Frederick MJ, Pickering CR, Bettegowda C, Chang K, Pickering CR, Bettegowda C, Chang K, Li RJ, Fakhry C, Xie TX, Zhang J, Wang J, Zhang N, El-Naggar AK, Jasser SA, et al. Exome sequencing of head and neck squamous cell carcinoma reveals inactivating mutations in NOTCH1. Science. 2011; 333:1154-1157.

102. Lawrence MS, Stojanov P, Mermel CH, Robinson JT, Garraway LA, Golub TR, Meyerson M, Gabriel SB, Lander ES, Getz G. Discovery and saturation analysis of cancer genes across 21 tumour types. Nature. 2014; 505:495-501.

103. Cao Y, Janssen EM, Duncan AW, Altman A, Billadeau DD, Abraham RT. Pleiotropic defects in TCR signaling in a Vav-1-null Jurkat T-cell line. EMBO J. 2000; 21:4809-4819.

104. Lai SY, Ziober AF, Lee MN, Cohen NA, Falls EM, Ziober BL. Activated Vav2 modulates cellular invasion through Rac1 and Cdc42 in oral squamous cell carcinoma. Oral Oncol. 2008; 44:683-688.
105. Patel V, Rosenfeldt HM, Lyons R, Servitja JM, Bustelo XR, Siroff M, Gutkind JS. Persistent activation of Rac1 in squamous carcinomas of the head and neck: evidence for an EGFR/Vav2 signaling axis involved in cell invasion. Carcinogenesis. 2007; 28:1145-1152.

106. Batson J, Maccarthy-Morrogh L, Archer A, Tanton H, Nobes CD. EphA receptors regulate prostate cancer cell dissemination through Vav2-RhoA mediated cell-cell repulsion. Biol Open. 2014; 3:453-462.

107. Hunter SG, Zhuang G, Brantley-Sieders D, Swat W, Cowan CW, Chen J. Essential role of Vav family guanine nucleotide exchange factors in EphA receptor-mediated angiogenesis. Mol Cell Biol. 2006; 26:4830-4842.

108. Dong Z, Liu Y, Lu S, Wang A, Lee K, Wang LH, Revelo M, Lu S. Vav3 oncogene is overexpressed and regulates cell growth and androgen receptor activity in human prostate cancer. Mol Endocrinol. 2006; 20:2315-2325.

109. Uen YH, Fang CL, Hseu YC, Shen PC, Yang HL, Wen KS, Hung ST, Wang LH, Lin KY. VAV3 oncogene expression in colorectal cancer: clinical aspects and functional characterization. Sci Rep. 2015; 5:9360.

110. Chen X, Chen SI, Liu XA, Zhou WB, Ma RR, Chen L. Vav3 oncogene is upregulated and a poor prognostic factor in breast cancer patients. Oncol Lett. 2015; 9:2143-2148.

111. Aguilar H, Urruticoechea A, Halonen P, Kiyotani K, Mushiroda T, Barril X, Serra-Musach J, Islam A, Caizzi L, Di Croce L, Nevedomskaya E, Zwart W, Bostner J, et al. VAV3 mediates resistance to breast cancer endocrine therapy. Breast Cancer Res. 2014; 16:R53.

112. Palomba G, Loi A, Porcu E, Cossu A, Zara I, Budroni M, Dei M, Lai S, Mulas A, Olmeo N, Ionta MT, Atzori F, Cuccuru G, et al. Genome-wide association study of susceptibility loci for breast cancer in Sardinian population. BMC Cancer. 2015; 15:383.

113. Kwon AY, Kim GI, Jeong JY, Song JY, Kwack KB, Lee C, Kang HY, Kim TH, Heo JH, An HJ. VAV3 Overexpressed in Cancer Stem Cells Is a Poor Prognostic Indicator in Ovarian Cancer Patients. Stem Cells Dev. 2015; 24:1521-1535.

114. Citterio C, Menacho-Marquez M, García-Escudero R, Larive RM, Barreiro O, Sánchez-Madrid F, Paramio JM, Bustelo XR. The rho exchange factors Vav2 and Vav3 control a lung metastasis-specific transcriptional program in breast cancer cells. Sci Signal. 2012; 115:5. ra71.

115. Razidlo GL, Magnine C, Sletten AC, Hurley RM, Almada LL, Fernandez-Zapico ME, Ji B, McNiven MA. Targeting pancreatic cancer metastasis by inhibition of Vav1, a driver of tumor cell invasion. Cancer Res. 2015; 75:2907-2915. 